\title{
Analysis of the Internal Electrical Characteristics of Electronic Power Transformers
}

\author{
Yang $\mathrm{Yi}^{*}$, Cheng-Xiong Mao ${ }^{*}$, Dan Wang ${ }^{\dagger}$, and Ji-Ming $\mathrm{Lu}^{* *}$ \\ *State Key Laboratory of Advanced Electromagnetic Engineering and Technology, Huazhong University of Science \\ and Technology, Wuhan, China \\ $\uparrow^{* * *}$ College of Electrical and Electronic Engineering, Huazhong University of Science and Technology, Wuhan, \\ China
}

\begin{abstract}
The modularized subunit of an electronic power transformer (EPT) is a series connection of two H-bridge voltage-source converters and a DC-DC converter with a high-frequency isolation transformer (HFIT). On the basis of cascading and paralleling the modularized subunits, EPT can be used in high-voltage and large-current applications in the power system. This paper discusses the steady state analysis of the modularized subunit of EPT. Theoretical analysis considers the influences of the two H-bridge voltage-source converters on the two sides of the DC-DC converter. We deduce the formulas of the theoretical calculation on the internal electrical characteristics of EPT (e.g., the voltages of the DC-bus capacitor and the primary side peak current of the HFIT). This paper provides guidance on the design and selection of EPT key elements (e.g., the DC-bus capacitors and HFIT). Experimental results are obtained from a single subunit of a laboratory model rated at $962 \mathrm{~V}, 15 \mathrm{kVA}$. All calculations, simulations, and experiments confirm the theoretical analysis of the subunit of EPT.
\end{abstract}

Key words: DC-bus voltage, Electronic power transformer (EPT), High-frequency isolation transformer (HFIT), Internal electrical characteristics of EPT, Peak current of HFIT

\section{INTRODUCTION}

The electronic power transformer (EPT) is a new form of power transformer that realizes the functions of traditional power transformers and performs power quality functions, such as power factor correction, voltage regulation, voltage sag and swell elimination, voltage flicker reduction, and protection capability, in the power system during fault situations [1]-[6]. EPT has garnered much interest because of its enormous potential in the field of smart grid. References [3]-[10] discussed the advantages of EPT when applied in AC/DC distribution grids. Many researchers have also paid considerable attention to the EPT topologies and control algorithms. References [9]-[13] discussed the cascaded

Manuscript received Jun. 19, 2012; revised May 21, 2013

Recommended for publication by Associate Editor Bor-Ren Lin.

${ }^{\dagger}$ Corresponding Author: wangdan@mail.hust.edu.cn

Tel: +86-27-87542669, Fax: +86-27-87542669, Huazhong University of Science and Technology.

* State Key Laboratory of Advanced Electromagnetic Engineering and Technology, Huazhong University of Science and Technology, China

${ }^{* *}$ College of Electrical and Electronic Engineering, Huazhong University of Science and Technology, China multilevel topology for high voltage and large-current applications. However, studying high voltage and large-current EPT applications in the power system requires further study. Therefore, realizing the experimental prototype of EPT is important.

The cascaded multilevel topology of EPT is well suited for high-voltage and large-current applications. This topology should be designed based on the idea of modularization in the engineering practice [12], [13] (Fig. 1). The modularized subunit of EPT consists of three main stages: a primary stage (H-bridge voltage-source converter (H-VSC)), DC-link stage (DC-DC) converter with high-frequency isolation transformer (HFIT), and secondary stage (H-VSC). The two $\mathrm{H}-\mathrm{VSC}$ realize the rectifier and inverter. The DC-link stage realizes the voltage level change and galvanic isolation. The DC-link stage is bidirectional (Fig. 2(a)) [14]. However, this stage can be designed as a unidirectional stage to reduce cost and to simplify the configuration if EPT is used only to power passive loads (Fig. 2(b)).

Each stage requires active switches, capacitors/inductors, or HFITs. These passive devices dominate the size and weight of the EPT. Therefore, the research on the design and 


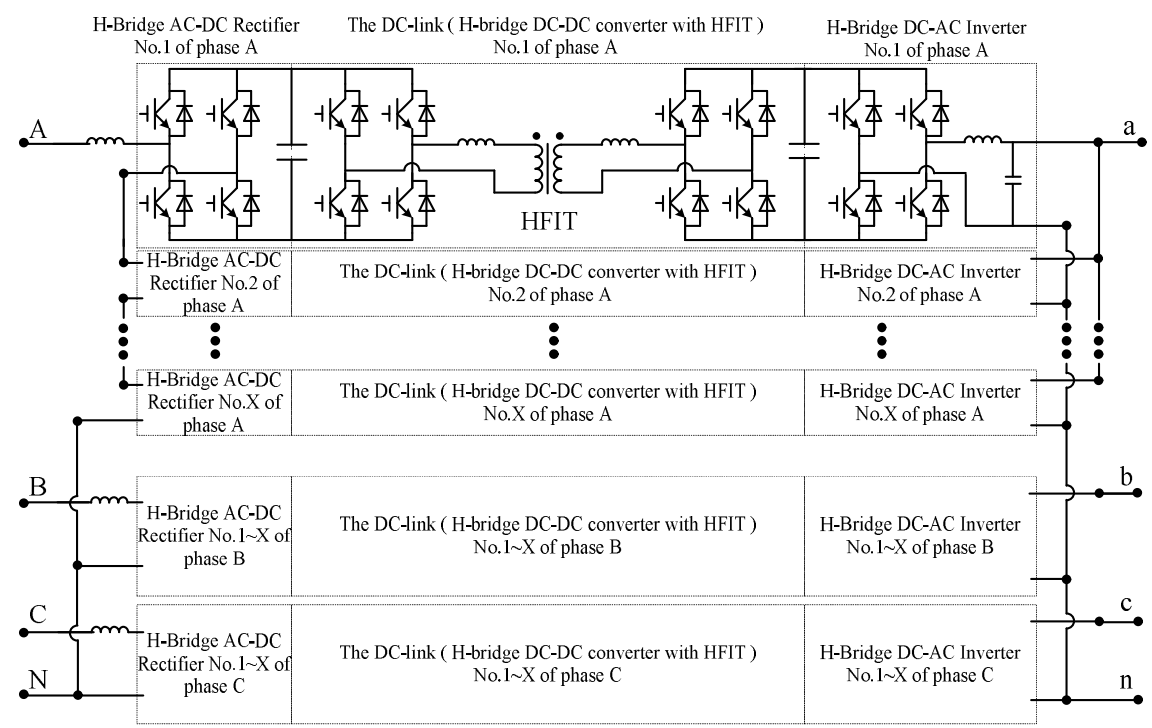

Fig. 1. Typical EPT structure.

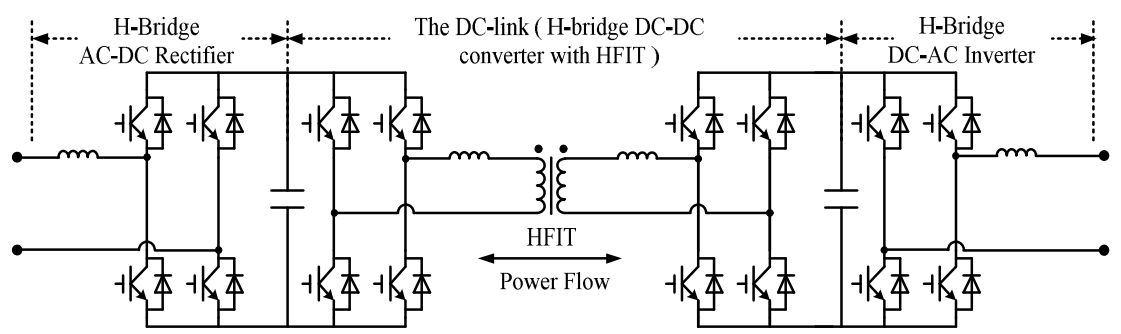

(a) Subunit using the bidirectional DC-link.

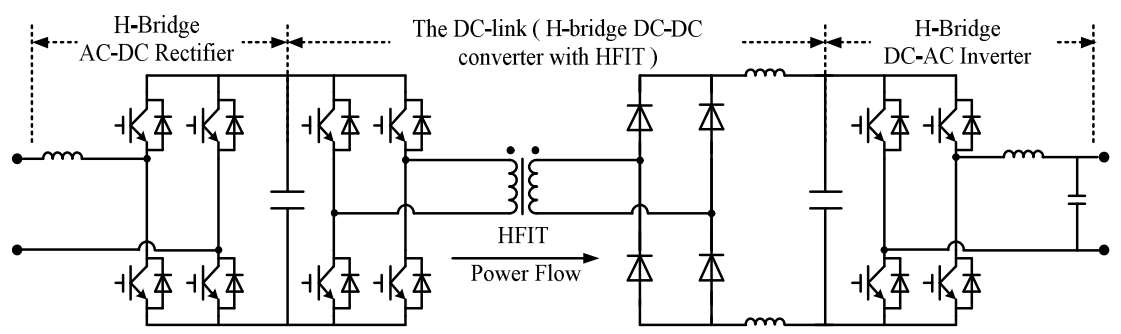

(b) Subunit using the unidirectional DC-link.

Fig. 2. Subunit of EPT.

selection methods of active and passive devices is important. A suitable design and selection of key elements (e.g., DC-bus capacitors and HFIT) can reduce the cost and size of EPT and guarantee safe operations. For example, if the rated current security margins of the capacitors and HFIT in the DC-link stage are large, the cost and size of the EPT may be unacceptable. If the security margins are too small, the EPT performance and service life may be degraded and shortened, respectively. However, few studies have focused on the key elements of EPT. Designing and selecting the key elements of EPT for power system applications is difficult because an exact and effective analysis of the internal characteristics of EPT is required.
Steady state analysis on the internal electrical characteristics of H-VSC and the DC-link stage has been proposed [14]-[18]. However, no analysis on the entire subunit of EPT (a series connection of two H-VSCs for the rectifier and inverter, as well as the DC-link stage (Fig. 2(b)) has been proposed. The respective analysis of the DC-link stage focuses on the converter itself and disregards the influence from the two H-VSCs. Analysis on the typical rectifier and inverter have also been proposed. However, the rectifier and inverter cannot be used directly on the analysis and calculation of the internal electrical characteristics of the entire EPT because the rectifier and inverter are only two stages of EPT. Design on fault tolerance should also be 


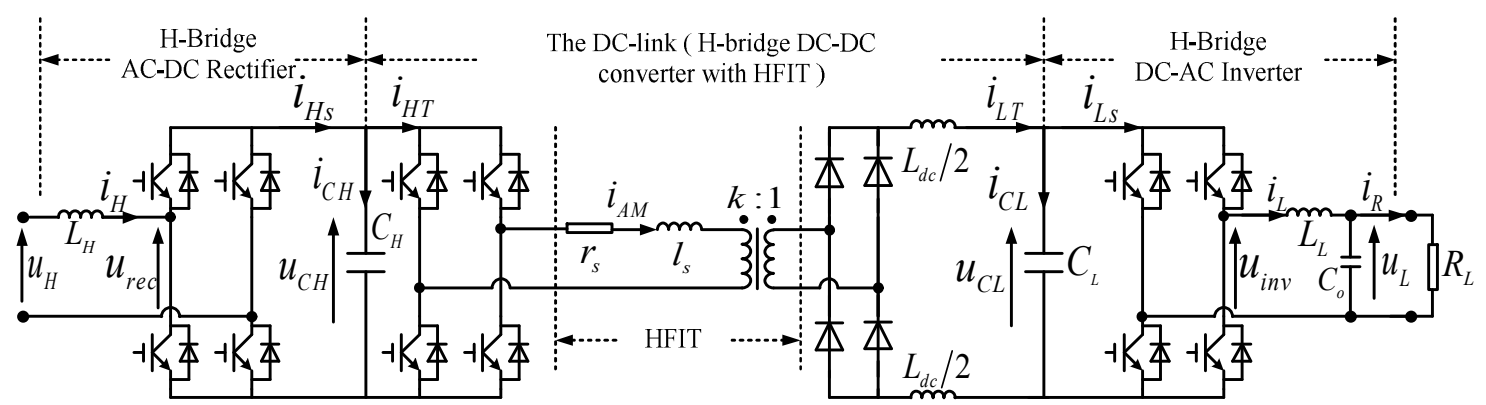

Fig. 3. Equivalent model of the subunit of EPT.

considered to increase EPT reliability. If one or more subunits fail, the static working point of normal subunits will change [13], [19]. The analysis in the current paper is different from that in the other papers. For instance, the amplitudes of the second harmonic voltages and two sides of the DC-bus at the DC-link stage influence each other closely. The HFIT peak current is decided by the two sides of the DC-bus voltages and HFIT parameters. Given the fault tolerance design, the internal external characteristics of EPT can be influenced if one or more subunits fail.

In this paper, we present a detailed analysis on the internal electrical characteristics of EPT. Section II describes both DC-bus voltages of the two sides and the HFIT peak current. Sections III and IV present the theoretical calculation, simulation and experimental results. Finally, Section IV presents the guidance on the design of the key elements.

\section{STEADy STATE ANALYSIS OF EPT}

Fig. 3 shows the equivalent model of the EPT subunit [20]. Currents $i_{H s}, i_{L s}, i_{H T}$, and $i_{L T}$ and voltages $u_{C H}$ and $u_{C L}$, which consist of the DC component and the second harmonic $\mathrm{AC}$ component, are defined as follows [16], [17]:

$$
\left\{\begin{array}{c}
i_{H s}=i_{H_{-} d c}+i_{H_{-} a c} \\
i_{L s}=i_{L s_{-} d c}+i_{L s_{-} a c} \\
i_{H T}=i_{H T_{-} d c}+i_{H T_{-} a c} \\
i_{L T}=i_{L T_{-} d c}+i_{L T_{-} a c} \\
u_{C H}=u_{C H_{-} d c}+u_{C H_{-} a c} \\
u_{C L}=u_{C L_{-} d c}+u_{C L_{-} a c}
\end{array},\right.
$$

where subscripts " $a c$ " and " $d c$ " denote the AC and DC components, respectively.

According to the Kirchhoff's current law and Fig. 3, we obtain the following:

$$
\begin{gathered}
\left\{\begin{array}{l}
i_{H_{s_{-}} d c}=i_{H T_{-} d c}, \\
i_{L s_{-} d c}=i_{L T_{-} d c}
\end{array}\right. \\
\left\{\begin{array}{l}
i_{H_{s_{-}} a c}=i_{C H}+i_{H T_{-} a c} \\
i_{L s_{-} a c}=-i_{C L}+i_{L T_{-} a c}
\end{array},\right.
\end{gathered}
$$

where $i_{C H}$ and $i_{C L}$ are the second $\mathrm{AC}$ current flowing through $C_{H}$ and $C_{L}$, respectively; $C_{H}$ and $C_{L}$ are also the capacitances of the DC-bus rectifier and inverter (Fig. 3).

Fig. 3 shows that the circuit of the H-bridge rectifier and inverter are almost the same and can be analyzed in the same manner. Assuming that the rectifier is working in the unity power factor, the rectifier AC voltage $\left(u_{r e c}\right)$, AC voltage $\left(u_{H}\right)$, and rectifier $\mathrm{AC}$ current $\left(i_{H}\right)$ are nearly in phase. Given that the frequency of the second harmonic AC currents $\left(i_{H s \_a c}\right)$ can double $i_{H}$, the phase angle of $i_{H_{-} a c}$ also doubles the phase angle $\left(\varphi_{H}\right)$ of the AC current $\left(i_{H}\right)[16][17]$ :

$$
i_{H s_{-} a c}=\frac{1}{2} i_{H} m_{H} \angle 2 \varphi_{H},
$$

where $m_{H}$ is the modulation index of the rectifier, and $\varphi_{H}$ is the phase angle of the AC voltage $\left(u_{H}\right)$.

Assuming that the load of the inverter is the pure resistor $\left(R_{L}\right)$, the inverter AC voltage $\left(u_{i n v}\right)$, AC voltage $\left(u_{L}\right)$, and load current $\left(i_{R}\right)$ are nearly in phase. The phase angle of the inverter AC currents $\left(i_{L}\right)$ is ahead of that of the load current $\left(i_{R}\right)$. The ahead phase angle is $\varphi_{\Delta}$, as shown below:

$$
\varphi_{\Delta}=\arctan \left(\omega R_{L} C_{o}\right),
$$

where $\omega$ is the fundamental angular frequency of the power system, $C_{o}$ is the capacitance of the inverter filter, and $L_{L}$ is the inductance of the inverter filter.

The second harmonic $\mathrm{AC}$ current $\left(i_{L_{-} a c}\right)$ is expressed as [16] [17] follows:

$$
i_{L s_{-} a c}=\frac{1}{2} i_{L} m_{L} \angle\left(2 \varphi_{L}+\varphi_{\Delta}\right),
$$

where $m_{L}$ is the modulation index of the inverter, and $\varphi_{L}$ is the phase angle of the AC voltage $\left(u_{L}\right)$.

Fig. 3 shows that the high-frequency H-bridge chopper is on the primary side of the HFIT and H-bridge diode rectifiers on the secondary side. The HFIT can be treated as a series connection of an equivalent $\mathrm{AC}$ resistance $\left(r_{s}\right)$, a leakage inductance $\left(l_{s}\right)$, and an ideal transformer (the transformer radio is $k: 1)$. The working frequency of the HFIT is the chopping frequency [20]. The equivalent AC resistance $\left(r_{s}\right)$ is estimated to be larger than the DC resistance of the windings because of the skin effect [18] [20]. The DC filter inductor $\left(L_{d c}\right)$ on the DC-bus of the H-bridge diode rectifier is represented in Fig. 3.

The total equivalent impedance of the HFIT and the 
DC-bus inductance is expressed as follows:

$$
Z_{H F I T+L d c}=\sqrt{r_{s}^{2}+\left(\omega_{H F} l_{s}\right)^{2}}+j k^{2} \omega^{\prime} L_{d c},
$$

where $\omega_{H F}$ is the chopping angular frequency of the DC-DC converter, and $\omega^{\prime}$ is the second harmonic angular frequency.

The primary and secondary sides of the second harmonic currents of the DC-bus are decided by the voltages of the two sides of the DC-bus. These sides can be expressed as follows:

$$
\left\{\begin{array}{rl}
i_{H T_{-} a c} & =\frac{u_{C H}-k u_{C L}}{Z_{H F I T+L d c}} \\
& =\frac{i_{C H} \angle \varphi_{C H}}{Z_{H F I T+L d c} \times j \omega^{\prime} C_{H}}-\frac{k i_{C L} \angle \varphi_{C L}}{Z_{H F I T+L d c} \times j \omega^{\prime} C_{L}} \\
i_{L T_{-} a c} & =\frac{\frac{1}{k} u_{C H}-u_{C L}}{\frac{1}{k^{2}} Z_{H F I T+L d c}} \\
= & \frac{k i_{C H} \angle \varphi_{C H}}{Z_{H F I T+L d c} \times j \omega^{\prime} C_{H}}-\frac{k^{2} i_{C L} \angle \varphi_{C L}}{Z_{H F I T+L d c} \times j \omega^{\prime} C_{L}}
\end{array},\right.
$$

where $\varphi_{C H}$ and $\varphi_{C L}$ are the phase angles of $i_{C H}$ and $i_{C L}$, respectively.

The HFIT currents $\left(i_{H T}\right.$ and $\left.i_{L T}\right)$ consist of the DC and second harmonic components. First, we analyze the second harmonic component.

By substituting Eqs. (4)-(8) in Eq. (3), we obtain the following:

$$
\left\{\begin{array}{l}
\frac{1}{2} i_{H} m_{H} \angle 2 \varphi_{H}= \\
i_{C H} \angle \varphi_{C H}+\frac{i_{C H} \angle \varphi_{C H}}{Z_{H F I T+L d c} \times j \omega^{\prime} C_{H}}-\frac{k i_{C L} \angle \varphi_{C L}}{Z_{H F I T+L d c} \times j \omega^{\prime} C_{L}} \\
\frac{1}{2} i_{L} m_{L} \angle\left(2 \varphi_{L}+\varphi_{\Delta}\right)= \\
-i_{C L} \angle \varphi_{C L}+\frac{k i_{C H} \angle \varphi_{C H}}{Z_{H F I T+L d c} \times j \omega^{\prime} C_{H}}-\frac{k^{2} i_{C L} \angle \varphi_{C L}}{Z_{H F I T+L d c} \times j \omega^{\prime} C_{L}}
\end{array} .\right.
$$

By solving Eq. (9), we can express the results as

$$
\left\{\begin{array}{l}
i_{C H} \angle \varphi_{C H}=\frac{a \times e+c \times d}{e \times b+f \times c} \\
i_{C L} \angle \varphi_{C L}=\frac{d \times b-a \times f}{e \times b+f \times c}
\end{array},\right.
$$

where the coefficients are defined as

$$
\begin{array}{lr}
a=\frac{1}{2} i_{H} m_{H} \angle 2 \varphi_{H} & b=1+\frac{1}{Z_{H F I T+L d c} \times j \omega^{\prime} C_{H}} \\
c=\frac{k}{Z_{H F I T+L d c} \times j \omega^{\prime} C_{L}} & d=\frac{1}{2} i_{L} m_{L} \angle\left(2 \varphi_{L}+\varphi_{\Delta}\right) \\
e=-1-\frac{k^{2}}{Z_{H F I T+L d c} \times j \omega^{\prime} C_{L}} & f=\frac{k}{Z_{H F I T+L d c} \times j \omega^{\prime} C_{H}} .
\end{array}
$$

According to Eq. (10), the second harmonic component voltages of the primary and secondary side DC-bus are expressed as follows $\left(u_{C H \_a c}\right.$ and $\left.u_{C L \_a c}\right)$ :

$$
\left\{\begin{array}{l}
u_{C H_{-} a c}=i_{C H} \angle \varphi_{C H} \times \frac{1}{j \omega^{\prime} C_{H}} \\
u_{C L_{-} a c}=i_{C L} \angle \varphi_{C L} \times \frac{1}{j \omega^{\prime} C_{L}}
\end{array} .\right.
$$

Second, we analyze the DC component. The rated power of the EPT subunit is defined as $P_{s}$. The currents $\left(i_{H s} d c\right.$ and $\left.i_{L s \_d c}\right)$ are expressed as

$$
\left\{\begin{array}{l}
i_{H s_{-} d c}=\frac{P_{s}}{u_{C H_{-} d c}} \\
i_{L s_{-} d c}=\frac{P_{s}}{u_{C L_{-} d c}} .
\end{array}\right.
$$

According to Eqs. (8) and (12), the primary side peak current of HFIT $\left(I_{\text {AMpeak }}\right)$ is the following:

$$
I_{\text {AMpeak }}=\left|i_{H T_{-} d c}\right|+\left|i_{H T_{-} a c}\right| .
$$

The frequency of the AC voltage $u_{\mathrm{H}}$ may change slowly in the range of $49.5 \mathrm{~Hz}$ to $50.5 \mathrm{~Hz}$, because the rectifier is directly connected to the power system. However, the frequency of the $\mathrm{AC}$ voltage in the inverter is constant as desired by the passive loads. The difference of the frequency between the $\mathrm{AC}$ voltages $\left(u_{H}\right.$ and $\left.u_{L}\right)$ can bring a variety of phase dissimilarities between the voltages. Eqs. (10), (11), and (13) describe the relationship among the three electrical characteristics and phase angles $\left(\varphi_{H}\right.$ and $\left.\varphi_{L}\right)$, which are in coefficients $a$ and $d$.

However, other research analyses have ignored the influence of the phase angles between the primary and secondary sides on the second harmonic component voltages. The peak current of HFIT is also neglected. The second harmonic component voltages of the primary and secondary sides of the DC-bus are expressed as follows $\left(u^{\prime}{ }_{C H} a c\right.$ and $\left.u_{C L \_a c}^{\prime}\right)[14][15]$ :

$$
\left\{\begin{array}{l}
u_{C H_{-} a c}^{\prime}=\frac{1}{2} i_{H} m_{H} \times \frac{1}{j \omega^{\prime} C_{H}} \\
u_{C L_{-} a c}^{\prime}=\frac{1}{2} i_{L} m_{L} \times \frac{1}{j \omega^{\prime} C_{L}}
\end{array} .\right.
$$

The fault tolerance design can increase the reliability of the EPT [13] [19]. If one or more EPT subunits fail and are successfully isolated, the EPT shall reach a new static working point with the normal running subunits, which must work reliably and safely in a faulted situation. However, the internal electrical characteristics of EPT in this faulted working situation should be compensated. Normal running subunits share the rated voltage of the cascaded side and the rated current of the parallel side. The $\mathrm{AC}$ current on the cascaded side and the AC voltage on the parallel side are considered to maintain invariability in a faulted working situation. The static working point of EPT can be calculated below.

The AC voltage $\left(u_{H(X-F)}\right)$ is on the cascaded side and is expressed as follows: 


$$
u_{H(X-F)}=\frac{u_{H} \times X}{(X-F)} .
$$

The DC voltage $\left(u_{C H \_d c(X-F)}\right)$ is on the cascaded side and is expressed as follows:

$$
u_{C H_{-} d c(X-F)}=\frac{u_{C H_{-} d c} \times X}{(X-F)} .
$$

The AC current $\left(i_{L(X-F)}\right)$ is on the parallel side and is expressed as follows:

$$
i_{L(X-F)}=\frac{i_{L} \times X}{(X-F)} .
$$

The DC voltage $\left(u_{C L \_c(X-F)}\right)$ is on the parallel side and is expressed as follows:

$$
u_{C L_{-} d c(X-F)}=\frac{u_{C L_{-} d c} \times X}{(X-F)} .
$$

The rated power of each normal running subunit $\left(P_{s(X-F)}\right)$ is given by the following:

$$
P_{s(X-F)}=\frac{P_{s} \times X}{(X-F)},
$$

where $X$ is the total number of the subunits of EPT per phase; $F$ is the number of the faulted subunits; $u_{H}, u_{C H}$, and $i_{L}$ are the $\mathrm{AC}$ and $\mathrm{DC}$ voltages on the cascaded side and the AC current on the parallel side in the working situation with all working subunits, respectively. We can logically calculate the subunit voltages and currents according to Eqs. (11) and (13).

On the basis of the formulas, the comparison between the calculation and simulation can prove the rationality of the theoretical calculation.

\section{COMPARISON OF THE THEORETICAL CALCULATION AND SIMULATION}

In this section, the simulations are conducted to demonstrate the above analysis on the system (see Fig. 2(b)). The frequency of $u_{L}$ is set to be similar to that of the AC voltage $\left(u_{H}\right)$. The phase difference $\left(\varphi_{L}-\varphi_{H}\right)$ between $u_{H}$ and $u_{L}$ is set from $0^{\circ}$ to $180^{\circ}$ every $10^{\circ}$. In this way, the theoretical calculation and simulation results are conducted to analyze the relationship between the parameters $\left(u_{C H_{-} a c}, u_{C L_{-} a c}\right.$, and $\left.I_{\text {AMpeak }}\right)$ and the phase difference $\left(\varphi_{L}-\varphi_{H}\right)$. The simulation is based on Matlab/Simulink.

The topology of the subunit for the theoretical calculation and simulation is shown in Fig. 2(b). The system parameters are listed in Table I.

\section{A. Theoretical Calculation Results}

On the basis of the formulas for theoretical calculation and parameters, the theoretical results are calculated according to Eqs. (11) and (13).

Fig. 4(a) shows the second harmonic component voltages of the primary side DC-bus $\left(u_{C_{-} \_c}\right)$ as polar coordinates, and the polar radius is defined as the amplitudes of $u_{C_{-} a c}$. Fig.

\section{TABLE I}

PARAMETERS OF THE THEORETICAL CALCULATION, Simulation AND PROTOTYPE SyStem.

\begin{tabular}{lll}
\hline \hline Symbol & \multicolumn{1}{c}{ Name } & Value \\
\hline$P_{s}$ & The rated power of the system & $15 \mathrm{~kW}$ \\
$u_{H}$ & The AC voltage of rectifier & $962 \times \sqrt{2} \mathrm{~V}$ \\
$u_{L}$ & The AC voltage of inverter & $220 \times \sqrt{2} \mathrm{~V}$ \\
$u_{C H} d c$ & The DC-bus voltage of rectifier & $1500 \mathrm{~V}$ \\
$u_{C L} d c$ & The DC-bus voltage of inverter & $380 \mathrm{~V}$ \\
$\omega^{\prime}$ & The 2-nd harmonic frequency & $628 \mathrm{rad} / \mathrm{s}$ \\
$\omega_{H F}$ & The working frequency of HFIT & $6280 \mathrm{rad} / \mathrm{s}$ \\
$L_{H}$ & The inductance of the rectifier & $20 \mathrm{mH}$ \\
$C_{H}$ & The capacitance of the primary side & $640 \mu \mathrm{F}$ \\
$L_{d c}$ & The filtering inductance of DC-bus & $0.6 \mathrm{mH}$ \\
$C_{L}$ & The capacitor of the secondary side & $0.0224 \mathrm{~F}$ \\
$L_{L}$ & The filtering inductance & $0.3 \mathrm{mH}$ \\
$C_{o}$ & The filtering capacitance & $100 \mu \mathrm{F}$ \\
$R_{L}$ & The load resistance & $3.2 \Omega$ \\
$r_{s}$ & The equivalent resistance of HFIT & $0.7 \Omega$ \\
$l_{s}$ & The leakage inductance of HFIT & $300 \mu \mathrm{H}$ \\
$k$ & The transformer ratio of HFIT & $3.9: 1$ \\
\hline \hline
\end{tabular}

on a single-phase $50 \mathrm{~Hz}$ base

4(b) shows the second harmonic component voltages of the secondary side DC-bus $\left(u_{C L_{-} a c}\right)$ as polar coordinates, and the polar radius is defined as the amplitudes of $u_{C L_{-} a c}$. Fig. 4(c) shows the primary side peak current of HFIT $\left(I_{\text {AMpeak }}\right)$ as polar coordinates, and the polar radius is defined as the peak current of HFIT $\left(I_{\text {AMpeak }}\right)$.

The voltage unit is volt $(\mathrm{V})$, the current unit is ampere (A), and the phase angle unit is degree $\left(^{\circ}\right)$. All voltages and currents in Fig. 4 are displayed as polar coordinates, and the polar angle is defined as double the phase difference $\left(2 \times\left(\varphi_{L}-\varphi_{H}\right)\right)$, which is from $0^{\circ}$ to $360^{\circ}$ every $20^{\circ}$ in the graphs.

\section{B. Simulation Results}

On the basis of the formulas for theoretical calculation and parameters described in Table I, the simulation results are presented in this section.

Fig. 4(a) shows the second harmonic component voltages of the primary side DC-bus $\left(u_{\mathrm{CH}_{-} a c}\right)$ as polar coordinates, and the polar radius is defined as the amplitudes of $u_{\mathrm{CH} a c}$. Fig. 4(b) shows the second harmonic component voltages of the secondary side DC-bus $\left(u_{C L_{-} a c}\right)$ as polar coordinates, and the polar radius is defined as the amplitudes of $u_{C L \_a c}$. Fig. 4(c) shows the primary side peak current of HFIT $\left(I_{\text {AMpeak }}\right)$ as polar coordinates, and the polar radius is defined as the peak current amplitudes of HFIT $\left(I_{\text {AMpeak }}\right)$.

The voltage unit is volt $(\mathrm{V})$, the current unit is ampere $(\mathrm{A})$, and the phase angle unit is degree $\left(^{\circ}\right)$. All voltages and currents in Fig. 4 are displayed as polar coordinates. The polar angle is defined as double the phase difference 


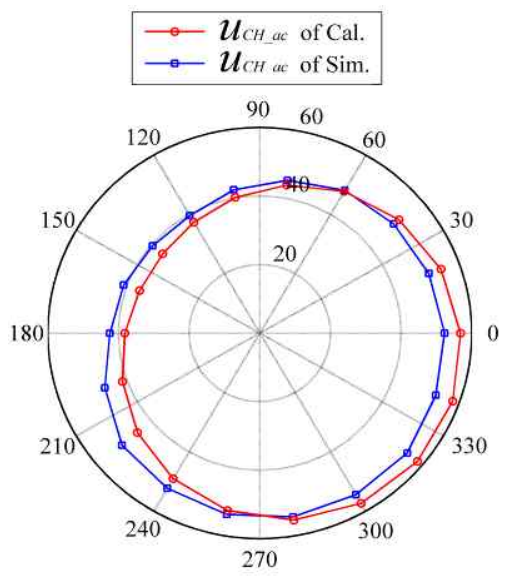

(a) Results of $u_{C H a c}(\mathrm{~V})$.

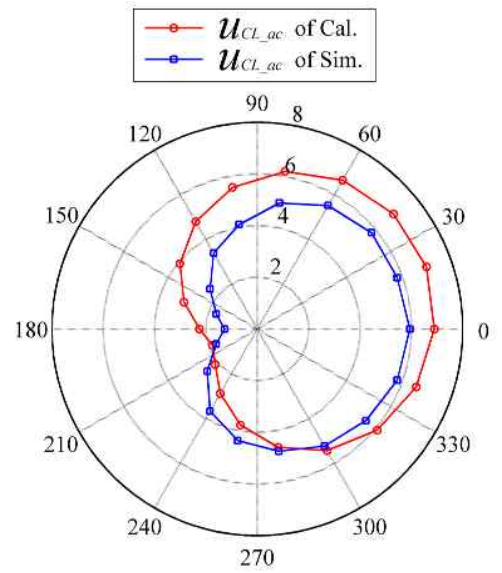

(b) Results of $u_{\text {ncloak }}(\mathrm{V})$.

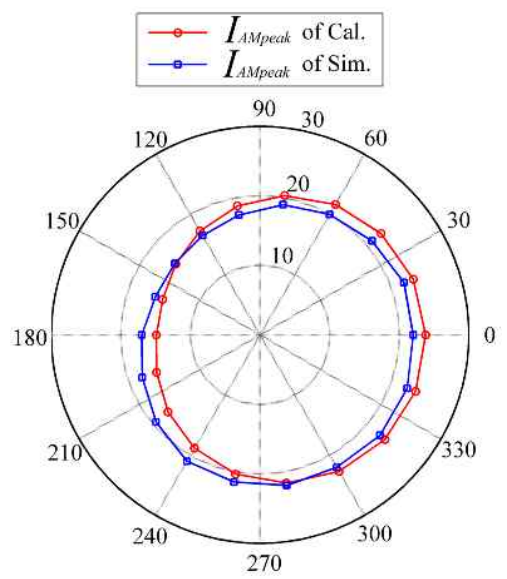

(c) Results of $I_{\text {AMpeak }}(\mathrm{A})$.

Fig. 4. Results of the theoretical calculation and simulation.

$\left(2 \times\left(\varphi_{L}-\varphi_{H}\right)\right)$, which is from $0^{\circ}$ to $360^{\circ}$ every $20^{\circ}$ in the graphs.

Fig. 4 shows the results of the theoretical calculation and simulation. The three groups of the curves in Figs. 4 (a)-(c) describe the relationship between electrical characteristics $\left(u_{C H \_a c}, u_{C L \_a c}\right.$, and $\left.I_{A M p e a k}\right)$ and polar angle. The voltages

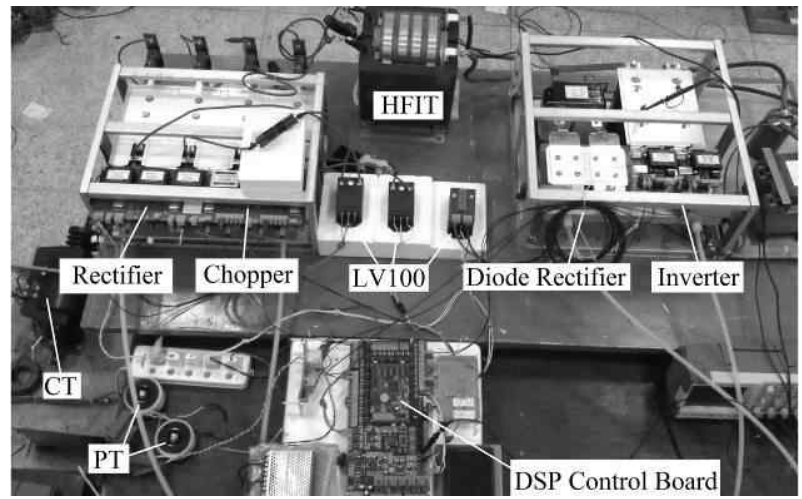

Fig. 5. The subunit of EPT in the laboratory.

$\left(u_{C H \_a c}\right.$ and $\left.u_{C L \_a c}\right)$ and the peak current $\left(I_{\text {AMpeak }}\right)$ are not constant, but they change with the polar angle from $0^{\circ}$ to $360^{\circ}$. The two curves in Figs. 4(a), 4(b), and 4(c) are all similar. The polar angle where $u_{C H a c}$ of calculation reaches the maximum point is almost similar to where $u_{C H a c}$ of simulation reaches the maximum point. The polar angle where $u_{C H \_a c}$ reaches the minimum point is also similar to where the $u_{C H a c}$ of the simulation reaches the minimum point. The curves of the theoretical calculation and simulation are also identical in Figs. 4(b) and 4(c). The similarity of the curve results can confirm the simulation.

According to Eq. (14) and Table I, the second harmonic component voltages of the primary and secondary side DC-bus are constant values, and $u_{C H a c}^{\prime}$ is about $54.9 \mathrm{~V}$, and $u_{C L a c}$ is about $6.9 \mathrm{~V}$. Compared with the calculation and simulation results shown in Fig. 4, the results according to Eq (14) ignored many detailed information. The theoretical calculation and simulation results according to Eqs. (11) and (13) include more details.

\section{THE EXPERIMENTAL RESULTS AND THE COMPARISON}

In this section, the experiments were carried out to prove rationality of the theoretical calculation and simulation of EPT internal electrical characteristics. Fig. 2(b) shows the circuit configuration of the experimental structure of EPT. Fig. 5 shows that the structure was built in the laboratory. The elements used in single subunit structure of EPT are tabulated in Table II.

Fig. 6 shows the digital control system configuration of the EPT single subunit on the basis of the digital controller with a digital signal processor (TMS320F28335). The closed-loop control strategy complete the following functions: (1) maintain the DC-bus voltage $\left(C_{H}\right)$ constant; (2) maintain the AC voltage $\left(u_{L}\right)$ constant; (3) correct the rectifier work in the unity power factor; (4) accurately set the phase difference between the $\mathrm{AC}$ voltages $\left(u_{H}\right.$ and $\left.u_{L}\right)$ from $0^{\circ}$ to $180^{\circ}$ every $10^{\circ}$. 


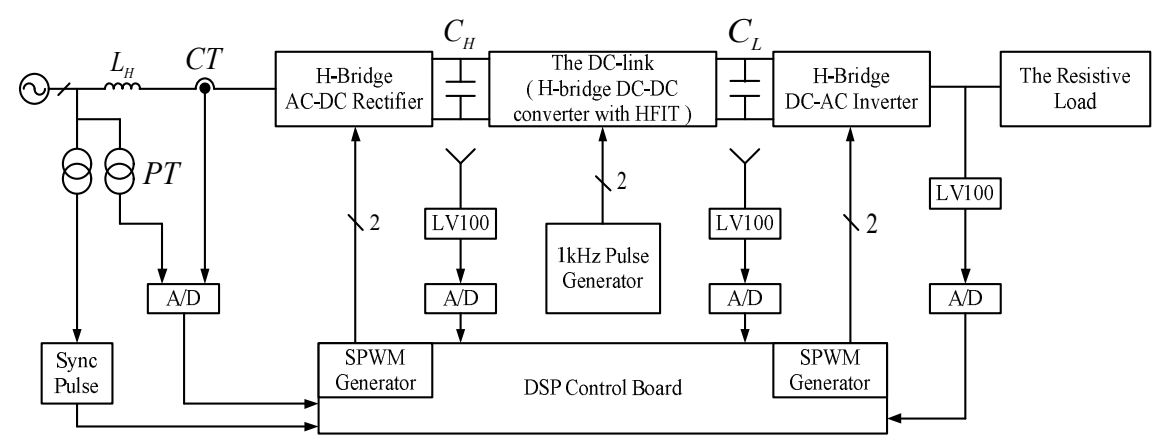

Fig. 6. Digital control system configuration.

TABLE II

The Elements of The Single Subunit Structure of EPT.

\begin{tabular}{llcc}
\hline \hline Names of elements & \multicolumn{1}{c}{ Type } & Manufacturer & Quantity \\
\hline IGBT of Rectifier & FF200R33KF2C & Infineon & 2 \\
IGBT of Inverter & FF450R12ME4 & Infineon & 2 \\
IGBT of the DC-DC Converter & FF200R33KF2C & Infineon & 2 \\
Diodes of the DC-DC Converter & MEO450-15DA & IXYS & 4 \\
Capacitors of the Primary Side DC-bus & SHP2000-160FS & EACO & 4 \\
Capacitors of the Secondary Side DC-bus & B43760B5568M3 & EPCOS & 4 \\
\hline \hline
\end{tabular}

\section{A. Experimental Results}

Fig. 7 shows the experimental waveforms of the EPT subunit at $15 \mathrm{kVA}$.

The experimental results are presented in this section on the basis of the single subunit of the laboratory model and the parameters described in Table I.

Fig. 8(a) shows the second harmonic component voltages of the primary side DC-bus $\left(u_{\mathrm{CH}_{a} a c}\right)$ as polar coordinates, and the polar radius is defined as the amplitudes of $u_{C_{-} a c}$. Fig. 8(b) shows the second harmonic component voltages of the secondary side DC-bus $\left(u_{C L_{a} a c}\right)$ as polar coordinates, and the polar radius is defined as the amplitudes of $u_{C L_{-} a c}$. Fig. 8(c) shows the primary side peak current of HFIT $\left(I_{\text {AMpeak }}\right)$ as polar coordinates, and the polar radius s defined as the peak current amplitudes of HFIT $\left(I_{\text {AMpeak }}\right)$.

The voltage unit is volt $(\mathrm{V})$, the current unit is ampere (A), and the phase angle unit is degree $\left({ }^{\circ}\right)$. All voltages and currents in Fig. 8 are displayed as polar coordinates. Polar angle was defined as double the phase difference $\left(2 \times\left(\varphi_{L}-\varphi_{H}\right)\right)$, which was from $0^{\circ}$ to $360^{\circ}$ every $20^{\circ}$ in the graph.

\section{B. Comparison of Results}

The results of the theoretical calculation, simulation, and experiments are all plotted in Fig. 8. Fig. 8 shows the internal electrical characteristics of EPT as a function of double the phase difference $\left(2 \times\left(\varphi_{L}-\varphi_{H}\right)\right)$ from $0^{\circ}$ to $360^{\circ}$.

Fig. 8(a) shows the second harmonic component voltages
$\left(u_{\mathrm{CH} \_a c}\right)$ of the primary side DC-bus as a function of double the phase difference $\left(2 \times\left(\varphi_{L}-\varphi_{H}\right)\right)$. The second harmonic component voltages $\left(u_{\mathrm{CH}_{-} a c}\right)$ reach a maximum value at the polar angle of about $300^{\circ}$ (Fig. 9(a)). The second harmonic component voltages $\left(u_{\mathrm{CH}_{a} a c}\right)$ reach a minimum value at the polar angle of about $120^{\circ}$, as shown in Fig. 9(b).

Fig. 8(b) shows the second harmonic component voltages $\left(u_{C L_{-} a c}\right)$ of the secondary side DC-bus as a function of double the phase difference $\left(2 \times\left(\varphi_{L}-\varphi_{H}\right)\right)$. The second harmonic component voltages $\left(u_{C L_{a} a c}\right)$ reach a maximum value at the polar angle of about $40^{\circ}$ (see Fig. 9(c)) and reach a minimum value at the polar angle of about $200^{\circ}$ (see Fig. 9(d)).

Fig. 8(c) shows the primary side peak current $\left(I_{\text {AMpeak }}\right)$ of HFIT as a function of double the phase difference $\left(2 \times\left(\varphi_{L}-\varphi_{H}\right)\right)$. The primary side peak current $\left(I_{\text {AMpeak }}\right)$ of HFIT reaches a maximum value at the polar angle of about $0^{\circ}$ (Fig. 9(e)). The primary side peak current $\left(I_{\text {AMpeak }}\right)$ of HFIT reached a minimum value at the polar angle of about $180^{\circ}$ (Fig. 9(f)).

According to the theoretical calculation, simulation, and experimental results, the voltages $\left(u_{C H_{a} a c}\right.$ and $\left.u_{C L_{a} a c}\right)$ and the peak current $\left(I_{\text {AMpeak }}\right)$ change with the phase angle. The three groups of the curves are similar (see Figs. 8(a)-(c)). Using the formulas we used above, similarity of the three groups of the curves confirmed the theoretical analysis of the EPT subunit.

According to Eq. (14) and Table I, the second harmonic component voltages of the primary and secondary side 


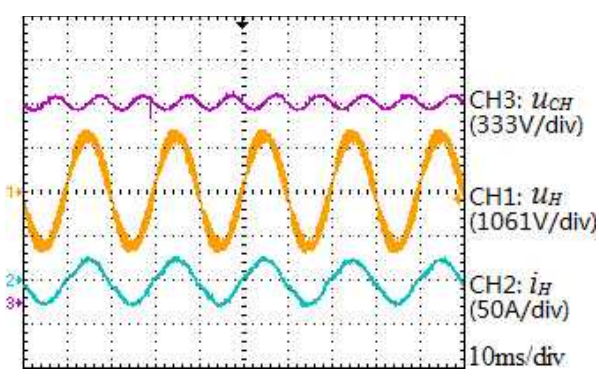

(a) Waveforms of $u_{H}, i_{H}$ and $u_{C H}$.

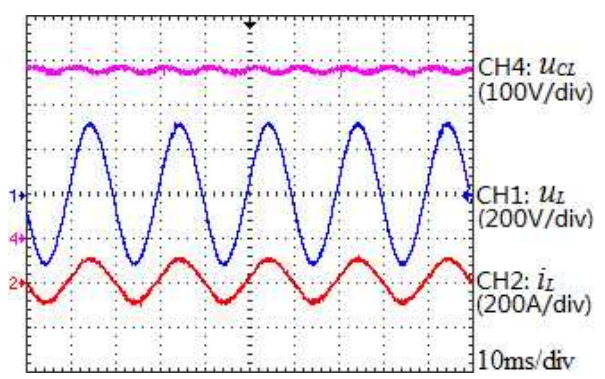

(b) Waveforms of $u_{L}, i_{L}$ and $u_{C L}$ of the inverter.

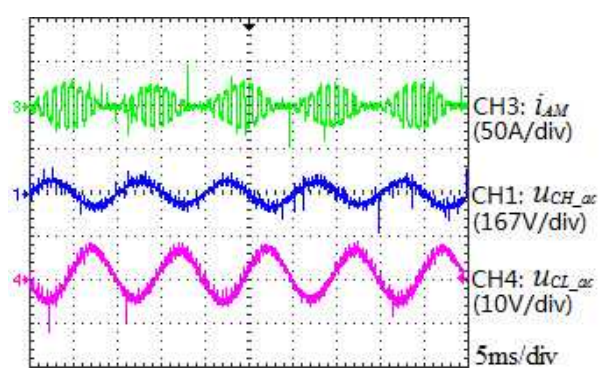

(c) Waveforms of $u_{C H \_a c}, u_{C L \_a c}$, and $i_{A M}$.

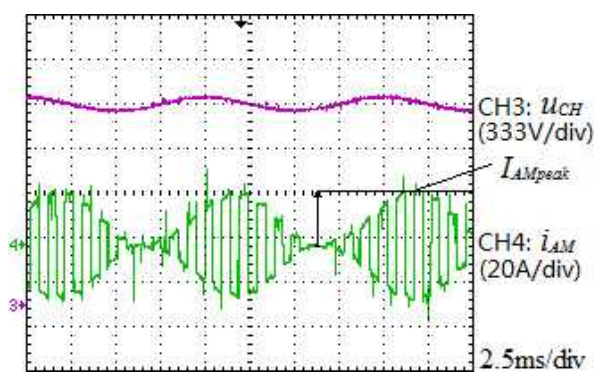

(d) Waveforms of $i_{A M}$ in detail.

Fig. 7. Experimental results of the EPT subunit working at the rated power when the phase angle is $0^{\circ}$.

DC-bus have been calculated in Section III. Compared with the results of the calculation, simulation, and experiment shown in Fig. 8, the results obtained by using Eq. (14) ignored many detailed information. The theoretical calculation and simulation results according to Eqs. (11) and (13) are more detailed and closer to the experimental results.

The difference among the three groups of the curves can be explained as follows:

- Theoretical analysis assumes all elements are ideal, and no losses are found on insulated-gate bipolar transistor

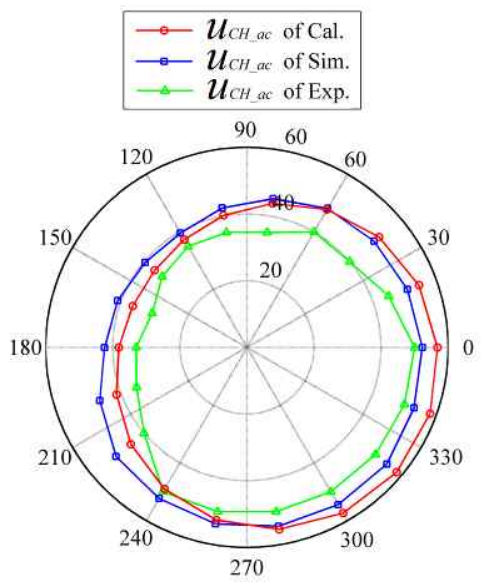

(a) Results of $u_{C H \_a c}(\mathrm{~V})$.

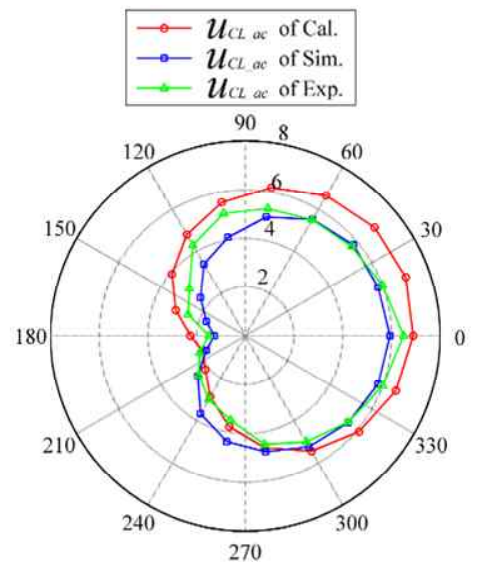

(b) Results of $u_{C L \_a c}(\mathrm{~V})$.

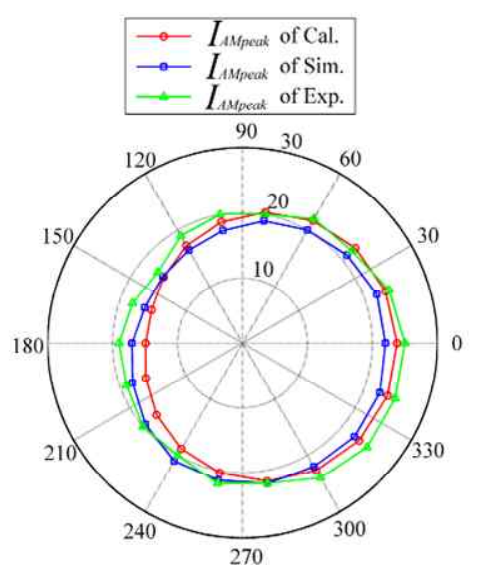

(c) Results of $I_{\text {AMpeak }}(\mathrm{A})$.

Fig. 8. Results of the theoretical calculation, simulation, and experiment.

(IGBT), diode, capacitors, and inductors. Loss of HFIT is equivalent to the equivalent of AC resistance $\left(r_{s}\right)$, which is a cursory estimate given the windings skin effect. The leakage inductance $\left(l_{s}\right)$ is also a cursory estimate. The IGBT, diode, capacitors, inductors, and HFIT are not 


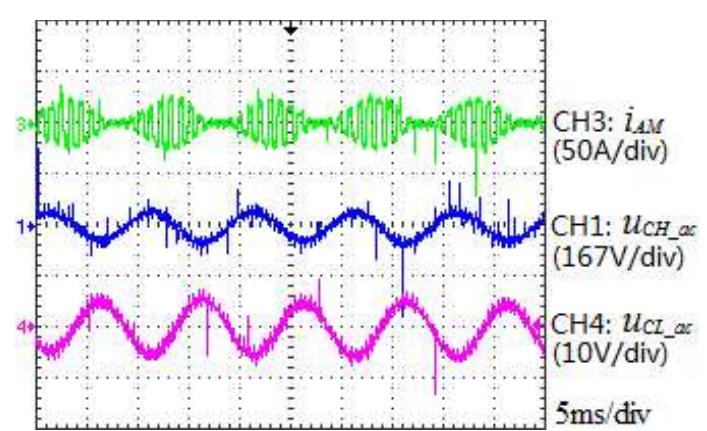

(a) $u_{C H \_a c}$ reaches a maximum value.

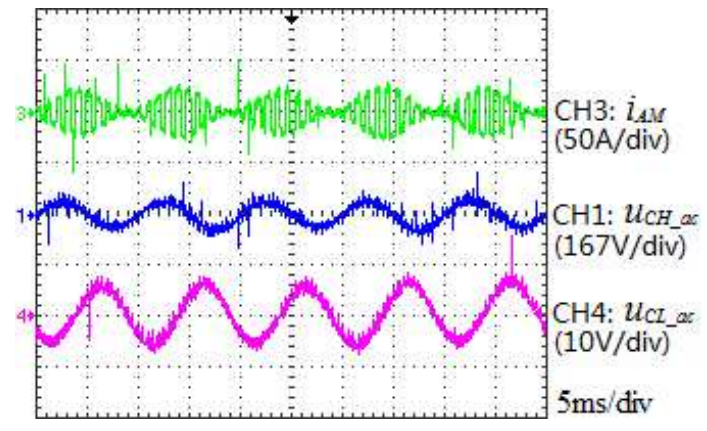

(c) $u_{C L \_a c}$ reaches a maximum value.

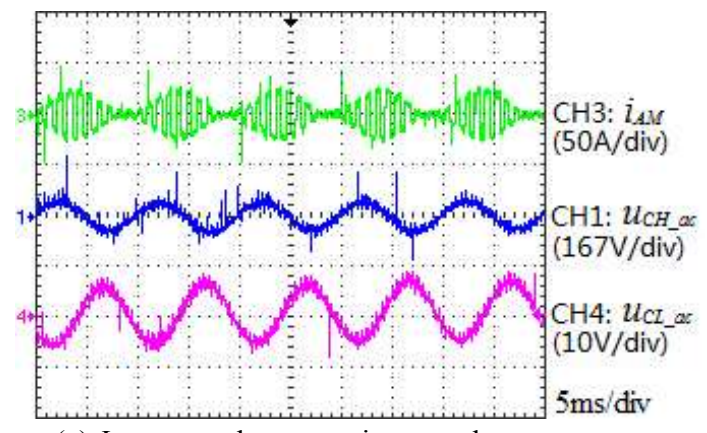

(e) $I_{\text {AMpeak }}$ reaches a maximum value.

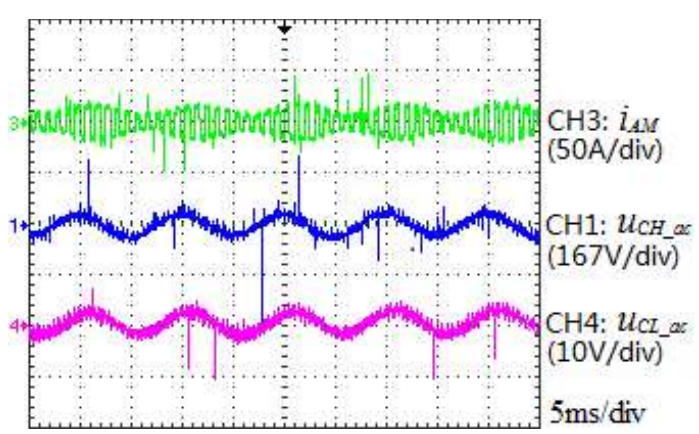

(b) $u_{C H a c}$ reaches a minimum value.

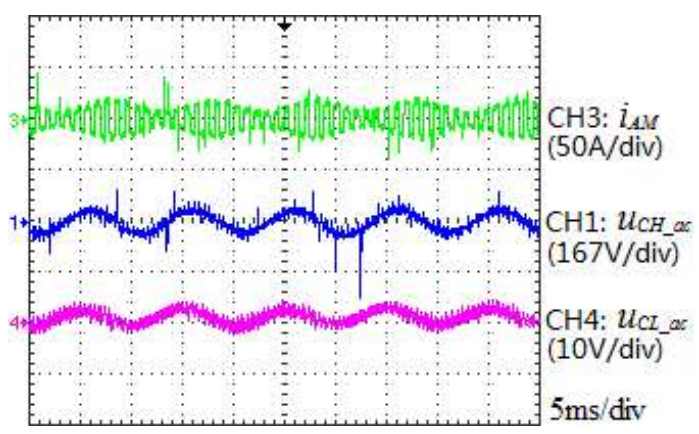

(d) $u_{C L a c}$ reaches a minimum value.

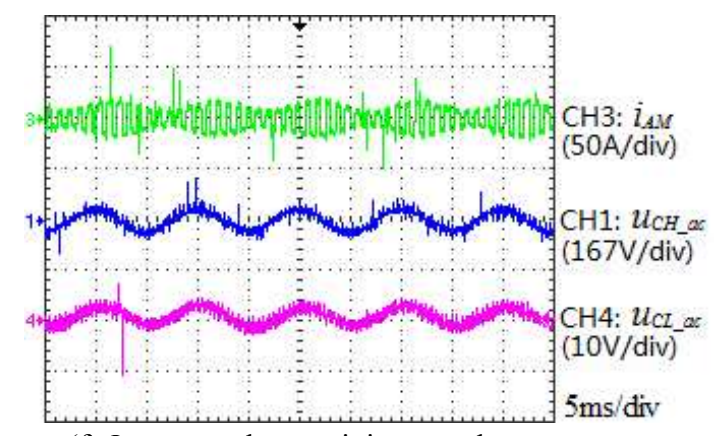

(f) $I_{\text {AMpeak }}$ reaches a minimum value.

Fig. 9. Waveforms of the experiment results.

treated as ideal in the simulation. The losses of all the elements are brought in by defining the corresponding parameters in the simulation models. The experiment is based on the subunit of EPT in the laboratory. The elements used in the experimental prototype of EPT are certainly not ideal.

- The parameters of the elements used in the single subunit of laboratory model are different more or less from that in the simulation and the calculation.

- The currents $i_{H s}, i_{L s}, i_{H T}$, and $i_{L T}$ consist of not only the DC component and the second harmonic component but also some higher harmonic components, which are all ignored in the theoretical analysis.

- The phase angle between $u_{r e c}$ and $u_{H}$, and the phase angle between $u_{i n v}$ and $u_{L}$ are also ignored in the theoretical analysis.

Therefore, the differences among the results of calculation, simulation, and experiment are existent but rational. Analyzing the subunit of EPT by using the formulas of the theoretical calculation is rational.

\section{Guidance on the Design of the Key Elements}

According to the analyses in this paper, guidance can be provided for designers when they select and design the key elements of EPT.

The second harmonic component voltages $\left(u_{\mathrm{CH}_{-} a c}\right.$ and $\left.u_{C L \_a c}\right)$ of the primary and secondary side DC-bus can be calculated in theory by using Eq. (11) to confirm the correctness of the capacitor selection. The security margins shall cover the values, which are determined by both the DC-bus capacitors and the system in the most severe conditions. The capacitors should be large enough to mitigate the second harmonic voltage and to limit the currents that flow through the capacitors to make them work normally. 
The capacitors should not be too large to limit capacitor cost and size.

The primary side peak current $\left(I_{\text {AMpeak }}\right)$ of HFIT can be calculated in theory by using Eq. (13) to confirm the correctness of the design of the rated current of HFIT. The security margins should cover the values determined by the system in the most severe conditions. The rated current of HFIT should be large enough to avoid overheating. However, the rated current should not be too large to limit cost and size.

On the basis of the fault tolerance design, the normal subunits of EPT must work safely even if some subunits are faulted. The security margins of the design should cover the values determined by the system in the most severe conditions. The values of $u_{C H_{-} a c}, u_{C L_{a} a c}$, and $I_{A M p e a k}$ can be calculated in theory according to Eqs. (11), (13), (15)-(19).

\section{CONCLUSIONS}

On the basis of the subunit of EPT, this paper presents the analysis method to calculate the internal electrical characteristics of EPT.

- The formulas of both the second harmonic component voltages $\left(u_{C H a c}\right.$ and $\left.u_{C L a c}\right)$ and the primary side peak current $\left(I_{\text {AMpeak }}\right)$ of HFIT are obtained in this paper.

- Compared with the results of the theoretical calculation, simulation, and experiment, analyzing the subunit of EPT by using the formulas in this paper is rational.

- The voltages $\left(u_{C H a c}\right.$ and $\left.u_{C C_{a} a c}\right)$ and the peak current $\left(I_{\text {AMpeak }}\right)$ change obviously with the polar angle. The voltages and currents will reach their maximum values at the specific polar angle in the faulted working situation. According to the maximum values, the guidance on the design and the selection of the key elements are provided for designers.

The single subunit structure of EPT has been built in a laboratory and in a $10 \mathrm{kV} / 400 \mathrm{~V}$ three-phase cascaded multilevel EPT (see Fig. 1), which is on the way of research and development. Further research on EPT will be conducted in the near future.

\section{ACKNOWLEDGMENT}

This work was supported by the Project of National Natural Science Foundation of China (51277083).

\section{REFERENCES}

[1] M. Kang, P. N. Enjeti, I. J. Pitel, "Analysis and design of electronic transformers for electric power distribution system," IEEE Trans. Power Electron., Vol. 14, No. 6, pp. 1133-1141, Nov. 1999.

[2] E. R. Ronan, S. D. Sudhoff, S. F. Glover, "A power electronic-based distribution transformer," IEEE Trans. Power Del., Vol. 17, No. 2, pp. 537-543, Apr. 2002.
[3] H. Rahmat-Allah, A. Mohammad and H. R. Mohammad, "Improving the dynamic performance of distribution electronic power transformers using sliding mode control," Journal of Power Electronics, Vol. 12, No. 1, pp. 145-156, Jan. 2012.

[4] D. Wang, C. X. Mao, J. M. Lu, J. P. He, and H. B. Liu, "Auto-balancing transformer based on power electronics," Electric Power Systems Research, Vol. 80, pp. 28-36, Jan. 2010.

[5] D. Wang, C. X. Mao, J. M. Lu, S. Fan, and F. Z. Peng, "Theory and application of distribution electronic power transformer," Electric Power systems Research, Vol. 77, No. 3-4, pp. 219-226, 2007.

[6] T. Zhao, G. Wang, S. Bhattacharya, A. Q. Huang. "Voltage and Power Balance Control for a Cascaded H-Bridge Converter-Based Solid-State Transformer," IEEE Trans. Power Electron., Vol. 28, No. 4, pp. 1523-1532, Apr. 2013.

[7] S. Falcones, R. Ayyanar, X. L. Mao, "A DC-DC multiport-converter-based solid-state transformer integrating distributed generation and storage," IEEE Trans. Power Electron., Vol. 28, No. 5, pp. 2192-2203, May. 2013.

[8] A. Q. Huang and J. Baliga, "FREEDM system: role of power electronics and power semiconductors in developing an energy internet," in Proc. 21st International Symposium on Power Semiconductor Devices \& IC's. ISPSD, pp. 9-12, 2009.

[9] J. J. Shi, W. Gou, H. Yuan, T. F. Zhao, A. Q. Huang, "Research on voltage and power balance control for cascaded modular solid-state transformer," IEEE Trans. Power Electron., Vol. 26, No. 4, pp. 1154-1166, Jul. 2011.

[10] H. Akagi, R. Kitada, "Control and design of a modular multilevel cascade BTB system using bidirectional isolated DC-DC converters," IEEE Trans. Power Electron., Vol. 26, No. 9, pp. 2457-2464, Sep. 2011.

[11] H. Fan and H. Li, "High-frequency transformer isolated bidirectional DC-DC converter modules with high efficiency over wide load range for $20 \mathrm{kVA}$ solid-state transformer," IEEE Trans. Power Electron., Vol. 26, pp. 3599-3608, Dec. 2011

[12] H. I. Eini, S. Farhangi, J. L. Schanen, M. K. Fard, “A modular power electronic transformer based on a cascaded H-bridge multilevel converter," Electric Power Systems Research, Vol. 79, pp. 1625-1637, 2009.

[13] Y. M. Park, H. S. Ryu, H. W. Lee, M. G. Jung, S. H. Lee, "Design of a cascaded h-bridge multilevel inverter based on power electronics building blocks and control for high performance," Journal of Power Electronics, Vol. 10, No. 3 , pp. 262-269, Mar. 2010.

[14] M. Kim, M. Rosekeit, S. Sul, and R. W. A. A. De Doncker, "A dual-phase-shift control strategy for dual-active-bridge DC-DC converter in wide voltage range," in Proc. IEEE 8th International Conference on Power Electronics and ECCE Asia (ICPE \& ECCE), pp. 364-371, 2011.

[15] F. Z. Peng, J. W. Mckeever, D. J. Adams, "A power line conditioner using cascade multilevel inverters for distribution systems," IEEE Trans. Ind. Appl., Vol. 34, No. 6, pp. 1293-1298, Nov./Dec 1998.

[16] S. Sirisukprasert, A. Q. Huang, J. S. Lai, "Modeling, analysis and control of cascaded-multilevel converter-based STATCOM," in Proc. IEEE Power Engineering Society General Meeting, Vol. 4, pp. 2561-2568, 2003.

[17] B. T. Zhang, Z. L. Nie, B. Li, L. P. Zhang, "Analysis of the DC-link capacitor voltage spectrum in the cascaded multilevel STATCOM," in Proc. IEEE International Conference on Industrial Technology, pp. 1-5, 2008. 
[18] I. Villar, U. Viscarret, I. Etxeberria-Otadui, A. Rufer. Global, "Loss evaluation methods for nonsinusoidally fed medium-frequency power transformers," IEEE Trans. Ind. Electron., Vol. 56, No. 10, pp. 4132-4140, Oct. 2009.

[19] H. I. Eini, S. Farhangi, J. L. Schanen, M. K. Fard, "A fault-tolerant control strategy for cascaded h-bridge multilevel rectifiers," Journal of Power Electronics, Vol. 10, No. 1, pp. 34-42, Jan. 2010.

[20] M. William, Flanagan, Handbook of transformer design applications, 2nd edition, McGraw-Hill, chap. 2, 1992.

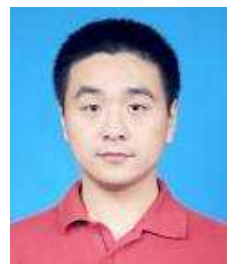

Yang Yi received his BS and MS degrees from the Department of Electrical Engineering, Huazhong University of Science and Technology (HUST), Hubei, China, in 2007 and 2009, respectively. His field of interest includes applications of high power electronic technology to power system. E-mail: yyceee@hust.edu.cn

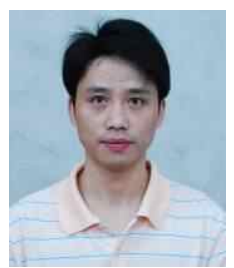

Dan Wang (M'07) received his BS, MS, and $\mathrm{PhD}$ degrees from the Department of Electrical Engineering, Huazhong University of Science and Technology (HUST), Hubei, China, in 1999, 2002, and 2006, respectively. He was a postdoctoral researcher from 2006 to 2008, sponsored by China Postdoctoral Science Foundation at the Department of Control Science and Engineering, HUST. From 2008 to 2009, he was a visiting research associate in the Department of Electrical and Computer Engineering at Michigan State University, United States. In 2008, he joined HUST. He is currently an associate professor at HUST. His research interest includes power system operations, control and power conditioning, and grid-connection of alternative energy sources. E-mail:wangdan@mail.hust.edu.cn

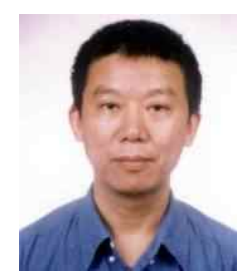

Ji-Ming Lu was born in Jiangsu, China, in 1956. He received his BS degree from the Department of Electrical Engineering, Shanghai Jiaotong University, Shanghai, China. He received his MS degree from the Department of Electrical Engineering, Huazhong University of Science and Technology (HUST), Hubei, China. In 1984, he joined the faculty of HUST. He is currently teaching at the Department of Electrical Engineering, HUST. His research includes excitation control based on microcomputer. E-mail: lujiming@mail.hust.edu.cn December 1994 to December 1995. He did research at Technische Universitaet Berlin from April 1996 to April 1997 under the support of Humboldt Foundation. He is currently a professor at HUST. His field of interest includes power system operation and control, the excitation control of synchronous generator, and applications of high power electronic technology to power system. E-mail: cxmao@mail.hust.edu.cn

Cheng-Xiong Mao (M'93-SM'08) received Technology (HUST), in 1984, 1987, and 1991, respectively. He was a visiting scholar at the University of Calgary, Canada, from January 1989 to January 1990. He also became a visiting scholar at Queen's University of Belfast from 